EPJ Web of Conferences 106, 03006 (2016)

DOI: $10.1051 /$ epjconf/201610603006

(C) Owned by the authors, published by EDP Sciences, 2016

\title{
ACS Algorithm in Discrete Ordinates for Pressure Vessel Dosimetry
}

\author{
William Walters ${ }^{\mathrm{a}}$ and Alireza Haghighat \\ Nuclear Engineering Program, Mechanical Engineering Department, Virginia Tech, 900N Glebe Rd, \\ Arlington, VA, USA
}

\begin{abstract}
The Adaptive Collision Source (ACS) method can solve the Linear Boltzmann Equation (LBE) more efficiently by adaptation of the angular quadrature order. This is similar to, and essentially an extension of, the first collision source method. Previously, the ACS methodology has been implemented into the TITAN discrete ordinates code, and has shown speedups of 2-4 on a simple test problem, with very little loss of accuracy (within a provided adaptive tolerance). This work examines the use of the ACS method for a more realistic problem: pressure vessel dosimetry with the VENUS-2 MOX-fuelled reactor dosimetry benchmark. The ACS method proved to be able to obtain accurate results while being approximately twice as efficient as using a constant quadrature in a standard source iteration scheme.
\end{abstract}

\section{Introduction}

Selection of an appropriate angular quadrature is one of the difficulties with the discrete ordinates method. Using a quadrature order that is too low will result in large errors from ray effects, while using an order too high greatly increases computation time.

A standard method to reduce ray effects and increase computation speed is to use a first collision source method [1, 2]. Our proposed methodology builds off the first-collision source method by separating not just the uncollided flux, but also the once-collided flux, twice collided flux, etc. At each transport iteration, we only solve for the i-th collided flux, and allow for the possibility of using different angular quadrature orders for each. We call this scheme the adaptive collision source (ACS) method [3,4]. This methodology has been implemented into the TITAN discrete ordinates transport code [5] and has shown excellent ability to improve the efficiency of simple transport calculations. In this paper, we discuss the ACS method as applied reactor dosimetry with the VENUS-2 problem.

The VENUS-2 MOX-fuelled reactor dosimetry benchmark [6, 7] was issued by the OECD/NEA based on the VENUS reactor owned and operated by SCK $\cdot \mathrm{CEN}$ in Belgium. The objective was to validate and compare nuclear data and transport codes for MOX-fuelled dosimetry calculations. In this work however, the goal is not to compare the ACS method to measured data, but to compare the

\footnotetext{
${ }^{a}$ Corresponding author: waltersw@vt.edu
}

This is an Open Access article distributed under the terms of the Creative Commons Attribution License 2.0, which permits unrestricted use, distribution, and reproduction in any medium, provided the original work is properly cited. 
ACS method to the standard source iteration method in TITAN. A comparison to measured data was performed in PENTRAN [8], which has a very similar algorithm to TITAN. Hence, if the ACS method can produce faster results than the standard source iteration in TITAN, similar accuracy to [8] can be achieved in a shorter time.

\section{Methodology}

This section is divided as follows: first, we will give some background and discuss the standard source iteration method to solve the Boltzmann equation, followed by the adaptive collision source method. Next, we examine our proposed method for adaptation of the angular quadrature order. Finally, we discuss the implementation into the TITAN transport code.

\subsection{Source Iteration}

The Linear Boltzmann Equation (LBE) for a fixed source can be written in operator form as:

$$
H \Psi=S \Psi+Q_{0} .
$$

Where, the streaming-collision operator $H$ and scattering operator $S$ are defined as:

$$
\begin{gathered}
H=\Omega \cdot \nabla+\sigma(\mathbf{r}, \mathrm{E}) \\
S=\int_{0}^{\infty} d E^{\prime} \int_{4 \pi} d \Omega \sigma_{s}\left(\boldsymbol{r}, E^{\prime} \rightarrow E, \Omega^{\prime} \cdot \Omega\right) .
\end{gathered}
$$

Both sides of Eq. (1) depend on $\Psi$ and cannot be solved directly, so in the standard source iteration method, an initial flux $\Psi^{(0)}$ (usually 0 ) is assumed, then the flux is calculated assuming a constant scattering source $S \Psi$, then the source is updated and the flux recalculated:

$$
H \Psi^{(\mathrm{i})}=S \Psi^{(\mathrm{i}-1)}+Q_{0} .
$$

Where, $\Psi^{(i)}$ is the flux after iteration $i$. After enough iterations, both sides of the equation will converge.

\subsection{Adaptive Collision Source (ACS) Method}

The ACS methodology [3,4] builds on the idea of the first-collision, but instead of splitting the flux up into uncollided flux and collided flux, total flux (denoted as $\Psi_{t, n}$ ) is expanded into the fluxes of different collision source order (i.e., 0 to $n$ ).

$$
\Psi_{t, n}=\Psi_{0}+\Psi_{1}+\Psi_{2}+\ldots+\Psi_{n}
$$

The series is truncated at the $n$ 'th collision and represents all particles that have undergone up to $n$ collisions. This is inserted into Eq. (1) to achieve the following formulation:

$$
H \Psi_{i}=Q_{i} \quad i=1,2, \ldots, n .
$$

For $i=0, Q_{0}$ is the independent source. For $i>0$, the $i$ 'th collision source $Q_{i}$ is defined as:

$$
Q_{i}=S \Psi_{i-1} .
$$

In the ACS method, each $\Psi_{i}$ is solved separately with discrete ordinates, then these are summed to get the total flux $\Psi_{t, n}$. For the calculation of each $\Psi_{i}$, a different angular quadrature can be used. By using different quadrature orders at different collision orders, a high order can be used for the first few collisions when needed before shifted to a lower order for the remaining scattering. 


\section{$15^{\text {th }}$ ISRD}

\subsection{Quadrature Order Adaptation Criteria}

In order to change quadrature for each $\Psi_{i}$, we need some criteria to decide when and how to do so. If we want to estimate the error produced by changing the quadrature order from $N$ to $N^{\prime}$ at a certain iteration, there are two types of error that we must consider. The first is the error in the flux of each iteration due to the quadrature change itself, which we will denote as quadrature error or $\epsilon_{\text {quad }}$. The second is the iterative error, denoted as $\epsilon_{i t e r}$ which represents the cumulative amount of flux that has yet to be calculated.

To estimate the error from changing angular quadrature, the angular flux from quadrature $N$ is projected onto the ordinates of quadrature $N^{\prime}$ and the scalar flux produced by each of these is compared. Further details on this projection can be found in Refs. 3 and 4. This leads to the following:

$$
\epsilon_{\text {quad }, i}=\frac{\phi_{i}^{N^{\prime}}-\phi_{i}^{N}}{\phi_{i}^{N}} .
$$

To calculate the iterative error, it is assumed that the flux is converging smoothly, to allow the following estimate to be reasonable:

$$
\phi_{i+1} \cong \Lambda \phi_{i} .
$$

Where, the constant $\Lambda$ can be interpreted as the spectral radius of the linear system. If Eq. (9) is valid, it can be shown that the error from stopping at iteration $i$ (instead of infinity) is a given by

$$
\epsilon_{i t e r}=\frac{\frac{\phi_{i}}{\phi_{t, i}}}{1-\frac{\phi_{i}}{\phi_{i-1}}} .
$$

The estimated total error is a combination of the iterative and quadrature errors:

$$
\epsilon_{\text {total }} \leq \epsilon_{\text {quad }} \cdot \epsilon_{\text {iter }}
$$

The quadrature adaptation process proceeds as follows. First, a list of quadrature sets is given (e.g., S16 and S8). For each energy group, the first iteration is performed using the highest quadrature. The error terms are calculated, and if the estimated error is lower than the tolerance, then the next lower quadrature set is used for the following iterations. The adaptation criteria is tested until the lowest quadrature set is reached.

\subsection{Implementation}

The ACS algorithm has been implemented into the TITAN discrete ordinates transport code. Since Eqs. $(6,7)$ are very similar to Eq. (4), the ACS method could be implemented into most general transport codes without extensive modification.

\section{Application}

\subsection{VENUS-2 Dosimetry Benchmark}

The VENUS-2 reactor horizontal description is given in Fig. 1. Vertically, the fuel length is $50 \mathrm{~cm}$, with reflector regions above and below. A full diagram is omitted for brevity, but the full description can be seen in the benchmark description [6].

The reactor core consists of 3 regions: an inner region (III) with $\mathrm{UO}_{2} 3.3$ (w/o) enriched, an outer region (IV) with $\mathrm{UO}_{2} 4.0$ (w/o) enriched and a 2.0/2.7 w/o MOX region (V). The reactor core is surrounded by steel and water regions. 


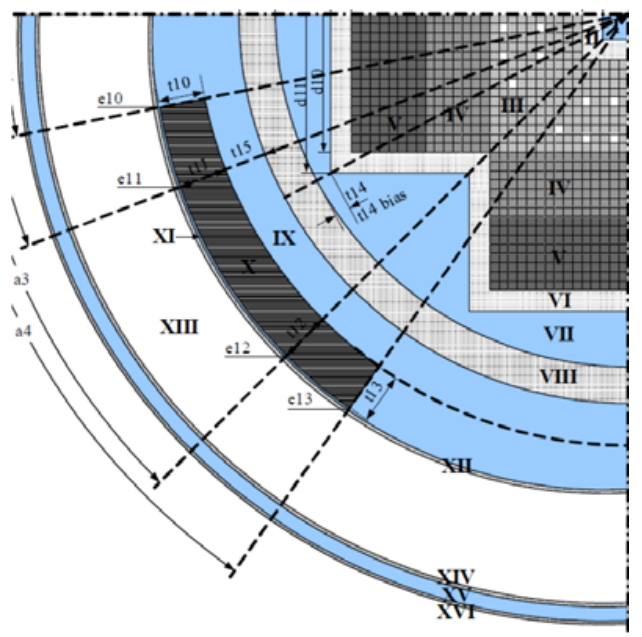

Figure 1. X-Y Diagram of VENUS-2 Core (Quarter).
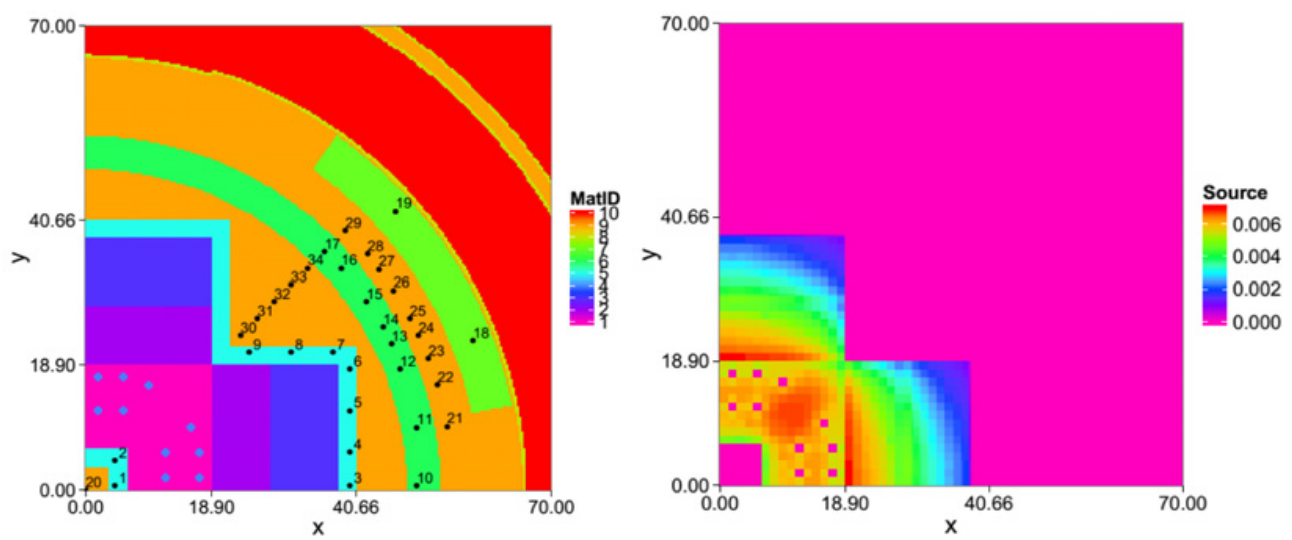

(a) material mesh distribution with detector positions. (b) source distribution.

Figure 2. TITAN model of VENUS-2.

The measured data in the benchmark are the reaction rates using ${ }^{58} \mathrm{Ni}(n, p),{ }^{115} \operatorname{In}\left(n, n^{\prime}\right),{ }^{103} \mathrm{Rh}\left(n, n^{\prime}\right)$, ${ }^{64} \mathrm{Zn}(n, p),{ }^{237} \mathrm{~Np}(n, f)$, and ${ }^{27} \mathrm{Al}(n, \alpha)$ dosimeters at 34 locations at the core midplane in regions I, II, and VI-X, which correspond to both steel and water locations.

\subsection{Calculation Parameters}

Using the benchmark information, a model was generated for TITAN, with a range of $70 \mathrm{~cm}$ to $168 \mathrm{~cm}$ on the $Z$ axis and 0 to $70 \mathrm{~cm}$ on the $X-Y$ plane. The mesh and materials for the core level are shown in Fig. 2a. The 34 detector locations are overlaid over the mesh distribution. Locations 1-19 indicate water locations, while locations 20-34 indicate steel regions. The source distribution used for the model is shown in Fig. 2b. Again, vertical diagrams are omitted for brevity.

Multi-group cross sections were derived from the 47-group BUGLE-96 [9] shielding cross-section library, based on the ENDF/B-VI data set. The fuel region was homogenized by volume fraction of fuel, 


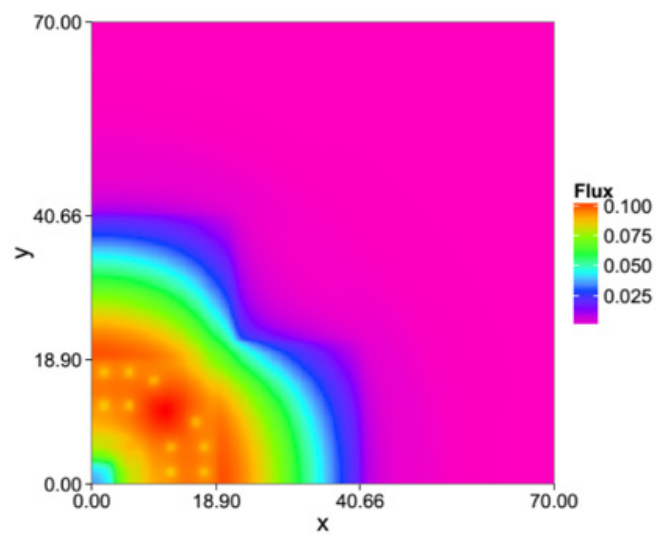

Figure 3. Reference flux for group $18(1.00-1.35 \mathrm{MeV})$.

clad and water. The dosimeter cross sections were obtained from BUGLE, although ${ }^{64} \mathrm{Zn}(\mathrm{n}$, p) data is not available so it was ignored in this analysis. Only the first 26 energy groups from BUGLE were used, which corresponds to 0.11 to $20 \mathrm{MeV}$, since below that the dosimeter cross sections are negligible.

The mesh size used was $0.315 \mathrm{~cm}$ in the $X / Y$ directions and $0.63 \mathrm{~cm}$ in the $Z$ direction, for a total of 557,583 spatial meshes. The linear-diamond scheme method is used for spatial differencing.

For angular quadrature, Legendre-Chebyschev (Pn-Tn) quadrature sets of order 4 to 16 (corresponding to 24-288 discrete ordinates) were used. Use of varying quadrature sets is required to test the effectiveness of the ACS method, since it involves adaptation of the angular quadrature.

Flux convergence was always set at 5.0E-4.

\subsection{Reference Results}

Reference 26-group flux results were obtained using the standard TITAN code with a S16 quadrature set. Fluxes for energy group $18(1.0-1.35 \mathrm{MeV})$ are shown in Fig. 3. As expected, the flux is highest in the core, dips in the center and in the Pyrex pins, and drops quickly outside of the core.

Using these fluxes, coupled with the dosimeter cross sections, reaction rates were obtained for every dosimeter location. Results for the ${ }^{27} \mathrm{Al}(n, \alpha)$ reaction are shown in Fig. 4 for all locations. This reaction was chosen since it is the highest threshold reaction of the dosimeter types, and thus should be most affected by quadrature (which is the subject of the ACS method).

Calculations were also performed using the standard TITAN code for lower quadrature orders (S4-S10). Relative errors compared to the S16 case for S4 and S10 are given in Fig. 5. As expected, the error decreases with quadrature order, and is highest in areas far from the source and dominated by streaming (i.e., regions IX and X).

To compare between cases, we consider the root mean square (RMS) relative error of all dosimeter locations and types. The maximum error is also considered. These errors, along with computation times for the TITAN calculations are given in Table 1. As expected, the errors consistently decrease while the computation time increases with the quadrature order. The "acceptable" quadrature level would depend on the required accuracy of the computation.

\subsection{ACS Results}

The same calculations were performed using the ACS-modified version of TITAN. For these calculations, the primary quadrature order was between 6 and 10, while the secondary quadrature order 


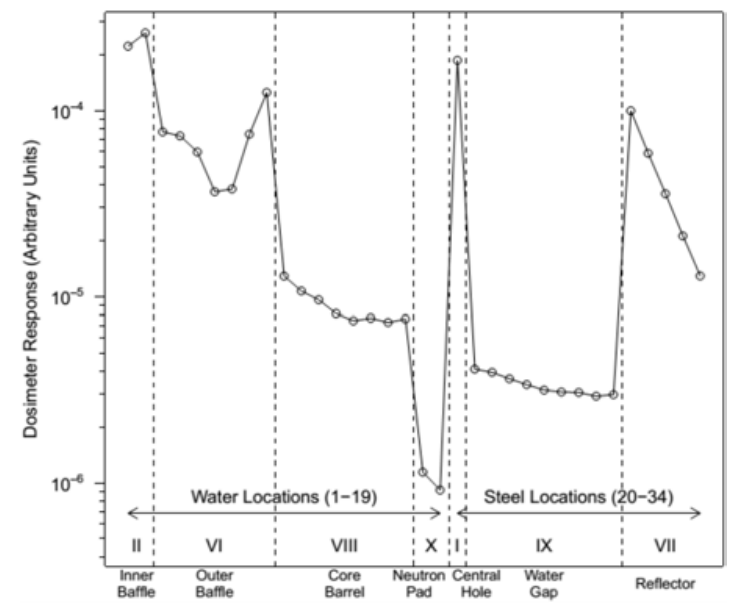

Figure 4. Reference reaction rates for ${ }^{27} \mathrm{Al}(n, \alpha)$ for all dosimeter locations.

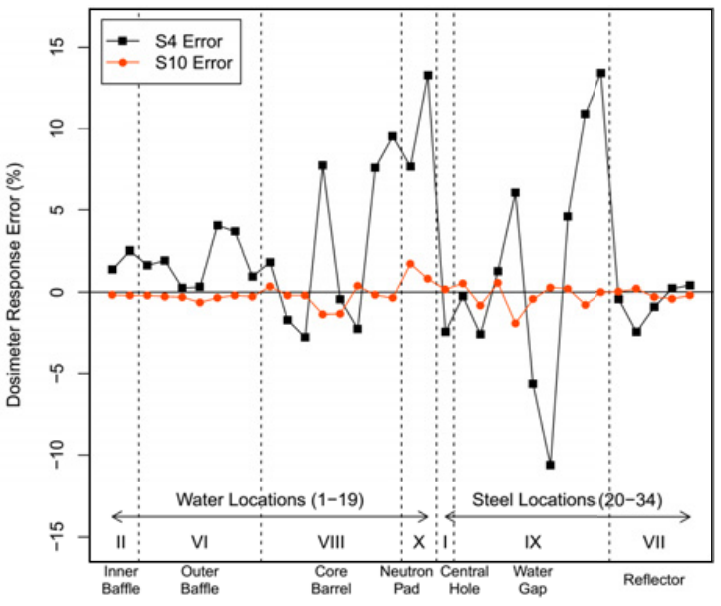

Figure 5. Reaction rate errors with lower quadrature for ${ }^{27} \mathrm{Al}(n, \alpha)$ at all dosimeter locations.

Table 1. Effect of quadrature order on standard calculations.

\begin{tabular}{|c|c|c|c|c|}
\hline $\begin{array}{c}\text { Quadrature } \\
\text { Order }\end{array}$ & $\begin{array}{c}\text { Computation } \\
\text { Time (min) }\end{array}$ & $\begin{array}{c}\text { RMS } \\
\text { Error }\end{array}$ & $\begin{array}{c}\text { Max } \\
\text { Error }\end{array}$ & $\begin{array}{c}\text { Speedup } \\
\text { (to S16) }\end{array}$ \\
\hline 16 & 1100.2 & - & - & 1.00 \\
\hline 10 & 452.5 & $0.37 \%$ & $1.91 \%$ & 2.43 \\
\hline 8 & 303.9 & $0.56 \%$ & $2.88 \%$ & 3.62 \\
\hline 6 & 187.3 & $0.93 \%$ & $4.22 \%$ & 5.87 \\
\hline 4 & 96.3 & $3.53 \%$ & $13.43 \%$ & 11.43 \\
\hline
\end{tabular}

was always 4. Calculation times and errors for ACS with S10/S4 is compared with standard TITAN in Table 2.

Note that as the adaptive tolerance increases, more iterations are performed at $\mathrm{S} 4$, the computation time goes down, and the error goes up. However, it is clear that the ACS method is much more efficient. For reasonable levels of tolerance, good speedups are obtained with very little increase in error. RMS 


\section{$15^{\text {th }}$ ISRD}

Table 2. Comparison of source iteration and ACS methods.

\begin{tabular}{|c|c|c|c|c|c|c|c|c|}
\hline $\begin{array}{c}\text { Quad. } \\
\text { Order }\end{array}$ & $\begin{array}{c}\text { ACS } \\
\text { Tol. } \\
(\epsilon)\end{array}$ & $\begin{array}{c}\# \\
\text { Iters } \\
(\text { S10) }\end{array}$ & $\begin{array}{c}\# \\
\text { Iters } \\
(\text { S4 })\end{array}$ & $\begin{array}{c}\text { Total } \\
\text { Iters }\end{array}$ & $\begin{array}{c}\text { RMS } \\
\text { Error } \\
(\%)\end{array}$ & $\begin{array}{c}\text { Max } \\
\text { Error } \\
(\%)\end{array}$ & $\begin{array}{c}\text { CPU } \\
\text { Time } \\
(\mathrm{s})\end{array}$ & Speedup \\
\hline \multicolumn{8}{|c|}{ Standard Source Iteration } \\
\hline 10 & - & 381 & - & 381 & $0.37 \%$ & $1.91 \%$ & 452.5 & 1.00 \\
\hline 4 & - & - & 393 & 393 & $3.53 \%$ & $13.43 \%$ & 96.3 & 4.70 \\
\hline \multicolumn{8}{|c|}{ ACS } \\
\hline $10 / 4$ & $10^{-3}$ & 234 & 158 & 392 & $0.43 \%$ & $2.35 \%$ & 296.3 & 1.53 \\
\hline $10 / 4$ & $10^{-2}$ & 111 & 281 & 392 & $0.59 \%$ & $2.38 \%$ & 188.1 & 2.41 \\
\hline $10 / 4$ & $10^{-1}$ & 28 & 365 & 393 & $2.03 \%$ & $6.63 \%$ & 115.9 & 3.90 \\
\hline
\end{tabular}

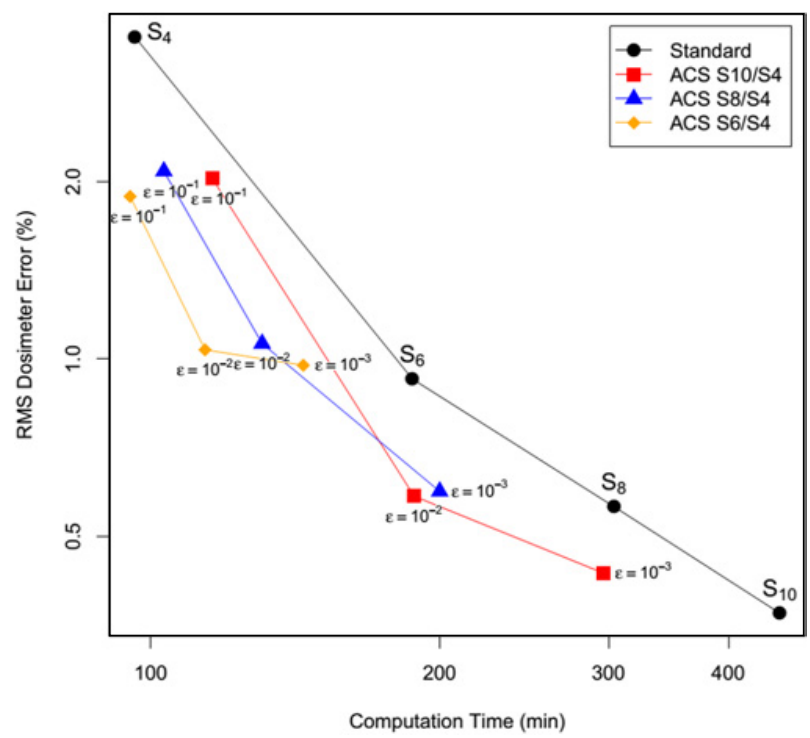

Figure 6. Comparison of source iteration and ACS methods for varying quadratures and adaptive tolerances.

error values and computation times for all cases are shown in Fig. 6. All cases offered a more efficient solution than the alternative of simply uniformly changing the quadrature. It is also noted that regardless of chosen parameters (quadrature and $\epsilon$ ), ACS performs better, so a priori knowledge of some optimal parameters is not required to obtain a speedup.

To get some insight on the details of an ACS calculation, one case is examined in more detail. Figure 7 shows the details of an ACS calculation for group 7 (4.97-6.07 MeV) with S10/S4 quadrature and $\epsilon=10^{-3}$. The calculations start using S10 and the estimated quadrature error (Eq. (8)) decreases over several iterations until reaching a constant value. The iterative error (Eq. (10)) decreases continuously after an initial period. The combined error (Eq. (11)) between these thus also decreases continuously, until it reaches the tolerance at iteration number 10. For subsequent iterations, S4 quadrature is used and no more quadrature error calculations can be performed. The shift in quadrature can be easily seen in the iteration time, which drops from over 8 seconds to below 2 seconds per iteration. The iterations are stopped when the iterative difference (i.e., $\phi_{i} / \phi_{t, i}$ ) reaches the flux tolerance (5.0E-4). 


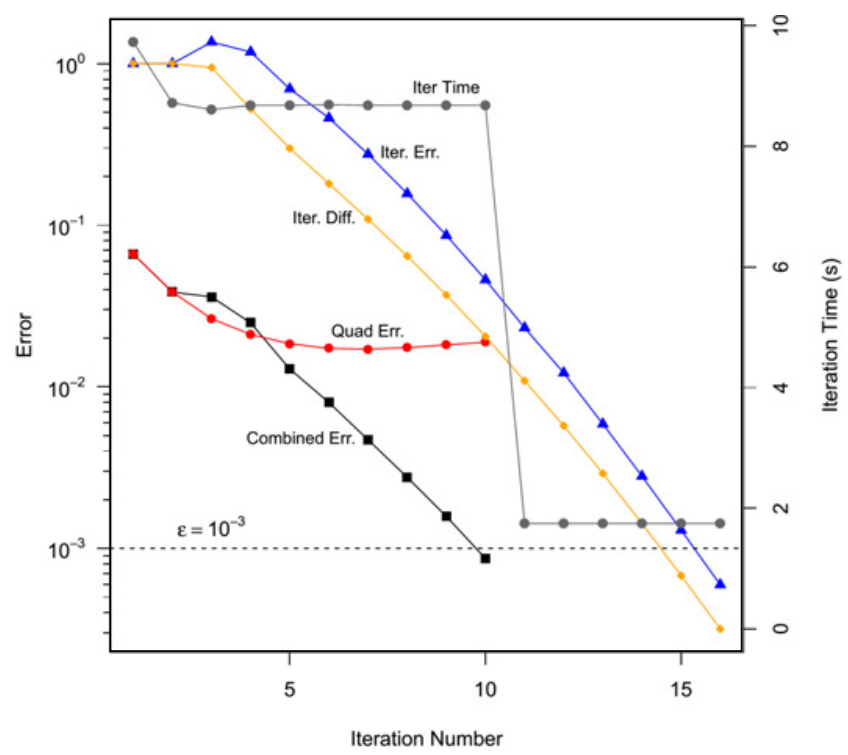

Figure 7. Estimated errors and computation times by iteration for group 7 (4.97-6.07 MeV) ACS calculation with S10/S4 quadrature and $\epsilon=10^{-3}$.

\section{Conclusions}

The adaptive collision source method has been shown to work well for on the VENUS-2 dosimetry benchmark, as compared to the standard source iteration method. As compared to the alternative of uniformly lowering the quadrature, ACS provides less error for the same gain in computation speed by approximately a factor of two.

\section{References}

[1] K.D. Lathrop, Nucl. Sci. Eng. 45, 255-268 (1971)

[2] R.A. Lillie, Proc. ANS RP\&S Top. Meet. (1998)

[3] W.J. Walters, A. Haghighat, Proc. Int. Conf. of Math. and Comp. (2013)

[4] W.J. Walters, A. Haghighat, Proc. Joint Int. Conf. Supercomputing in Nucl. Appl. + MC (2013)

[5] C. Yi, A. Haghighat, Nucl. Sci. \& Eng. 164, 221-247 (2010)

[6] C.Y. Han, C.H. Shin, H.C. Kim, J.K. Kim, N. Messaoudi, B.C. Na, Nuclear Energy Agency, NEA/NSC/DOC 22 (2005)

[7] G. Longoni, A. Haghighat, C. Yi, G.E. Sjoden, J. ASTM Int. 3 (2006)

[8] A. Haghighat, H.A. Abderrahim, G.E. Sjoden, Reactor Dosimetry ASTM STP 1398 (1999)

[9] J.E. White, D.T. Ingersoll, C.O. Slater, R.W. Roussin, Proc. ANS RP\&S. Top. Meet. (1996) 